ARTIGO

\title{
INCIDÊNCIA E FATORES ASSOCIADOS À OBESIDADE SARCOPÊNICA EM IDOSOS: UMA REVISÃO
}

\section{INCIDENDE AND FACTORS ASSOCIATED WITH SARCOPENIC OBESITY IN ELDERLY: A REVIEW}

\author{
Jéssica Bianca Alves de Sousa ${ }^{1}$ \\ Curso de Graduação em Nutrição - Centro de Ciências Biológicas e da Saúde - \\ Universidade Presbiteriana Mackenzie, São Paulo - SP, Brasil. \\ https://orcid.org/orcid-search/search?orcid=0000-0002-6224-9333 \\ jessicab.alves.sousa@gmail.com \\ Marcela Pereira Rodrigues ${ }^{2}$ \\ Curso de Graduação em Nutrição - Centro de Ciências Biológicas e da Saúde - \\ Universidade Presbiteriana Mackenzie, São Paulo - SP, Brasil. \\ https://orcid.org/orcid-search/search?orcid=0000-0003-4635-6953 \\ rodriguesalecram@gmail.com \\ João Orlando Jardim de Figueiredo ${ }^{3}$ \\ Curso de Graduação em Nutrição - Centro de Ciências Biológicas e da Saúde - \\ Universidade Presbiteriana Mackenzie, São Paulo - SP, Brasil. \\ https://orcid.org/0000-0002-5951-8846 \\ joaojfigueiredo19@gmail.com \\ Profa. Orientadora Dra. Daniela Maria Alves Chaud ${ }^{4}$ \\ Prof. Adjunto - Centro de Ciências Biológicas e da Saúde - Universidade Presbiteriana \\ Mackenzie, São Paulo - SP, Brasil. \\ Universidade Presbiteriana Mackenzie, São Paulo - SP \\ https://orcid.org/0000-0001-7405-8801 \\ daniela.chaud@mackenzie.br
}

\begin{abstract}
'Elaboração do projeto, levantamento bibliográfico, elaboração de todos os itens do artigo e formatação deste.

${ }^{2}$ Colaboração em todos os itens do trabalho e incremento do levantamento bibliográfico, da discussão e da conclusão. Auxílio na elaboração das sugestões dos avaliadores.

${ }^{3}$ Colaboração em todos os itens do trabalho, definição dos objetivos, elaboração da introdução, discussão e conclusão. Auxílio na elaboração das sugestões dos avaliadores. ${ }^{4}$ Orientação em todas as etapas do trabalho, correções preliminares, intermediárias e finais, acompanhamento da incorporação das sugestões dos avaliadores pelos demais autores.
\end{abstract}

\section{RESUMO}

O objetivo desta revisão foi analisar os dados na literatura científica referentes a incidência de obesidade sarcopênica (OS) em idosos, e suas possíveis associações. Trata-se de uma revisão de artigos nacionais publicados entre
2015 e 2019 nas bases de dados Scientific Electronic Library Online (SciELO), Biblioteca Virtual em Saúde (BVS) e Google Acadêmico. Observou-se um aumento na prevalência de OS, cuja a predominância é maior em mulheres e em indivíduos com idade a partir 
da sétima e oitava década. Além disso, contatou-se que a OS está associada ao desenvolvimento de fragilidade, mobilidade reduzida, e síndromes metabólicas e que idosos insuficientemente ativos possuem maior risco de desenvolver. A prática de atividade física e boa alimentação são fatores essenciais para prevenção e tratamento da OS.

Palavras-chave:

Envelhecimento.

Obesidade. Sarcopenia.

\section{ABSTRACT}

The objective of this review was to analyze data in the scientific literature regarding the incidence of sarcopenic obesity (SO) in the elderly and their possible associations. This is a review of national articles published between 2015 and 2019 in the databases Scientific Electronic Library Online (SciELO), Virtual Health Library - Brazil and Google. There was an increase in the prevalence of SO, whose prevalence was even higher in women and in individuals belonging in the seventh and eighth decades.

In addition, it has been reported that SO is associated with the development of frailty, reduced mobility, and metabolic syndromes and that insufficiently active elders are more likely to develop the syndrome. In short, the practice of physical activity and good nutrition are essential factors for the prevention and treatment of sarcopenic obesity.

Keywords: Aging. Obesity. Sarcopenia.

\section{INTRODUÇÃO}

Nos últimos anos pôde-se observar alterações na situação socioeconômica e de saúde, resultando na transição demográfica no Brasil. Tal transição engloba o aumento da expectativa de vida e consequentemente 0 aumento da população idosa (FERREIRA et al., 2010). São considerados idosos os indivíduos com idade maior ou igual a 60 anos. O envelhecimento está associado ao acúmulo de uma grande variedade de danos celulares, gerando: perda gradual nas reservas fisiológicas; aumento do risco doenças; declínio geral na capacidade intrínseca - composto de todas as capacidades físicas e mentais - e capacidade funcional do indivíduo (ORGANIZAÇÃO MUNDIAL DA SAÚDE, 2015).

Entre as afecções que acometem os indivíduos idosos estão a sarcopenia, obesidade sarcopênica (OS) e obesidade osteosarcopênica (OOS). A sarcopenia define-se por uma diminuição do tecido muscular como um resultado da redução do uso ou inutilização deste tecido. Trata-se de uma causa importante de incapacidade, dependência e fragilidade na população idosa (CRUZ-JENTOFTH et al., 2010; SAKUMA; YAMAGUCHI, 2013).

Quando a sarcopenia está associada ao excesso de gordura corporal, passa a denominar-se OS. Já a OOS engloba além da sarcopenia e obesidade, a osteopenia/osteoporose. Esta pode resultar em um quadro ainda mais agravante para redução do desempenho físico, risco de quedas, fraturas, hospitalizações e incapacidade funcional (SANTOS, 2018). Estima-se que a prevalência de OS seja entre $2 \%$ e $10 \%$ em idosos de ambos os sexos, com maiores valores percentuais também observados em idosos com idade igual ou superior a 80 anos (SANTOS et al., 2018). 
A etiologia da OS inclui interações entre os fatores causais da sarcopenia e da obesidade, como a redução progressiva no gasto energético total resultante do decréscimo na atividade física e taxa metabólica basal, nutrição inadequada e inflamação por meio da produção de citocinas pró-inflamatórias pelo tecido adiposo (SANTOS et al., 2017a; STENHOLM et al., 2008). O impacto negativo da OS sobre a qualidade de vida de idosos tem se tornado a principal preocupação dos profissionais da saúde, devido ao potencial prejuízo no desempenho motor e na capacidade funcional causada pela síndrome. Além disso, são fatores de risco para agravos cardiometabólicos, quedas, fragilidade e inclusive mortalidade (SIQUEIRA; GOBBO, 2015).

Idosos com OS e inflamatória crônica são mais suscetíveis a presença de síndrome metabólica, este fato é preocupante tendo em vista as consequências deletérias que essas condições clínicas podem repercutir na qualidade de vida desse grupo populacional (ROEDIGER et al., 2017). No entanto, devese levar em conta que tais agravos estão ligados diretamente a fatores comportamentais modificáveis, como a prática de atividade física e o tempo sedentário. Estudos mostram que indivíduos idosos com maior nível de atividade física apresentam reduções de gordura corporal e menor comprometimento da massa e força muscular (BANN et al., 2014; SANTOS, 2018).
Desta forma, o objetivo desta revisão foi analisar os dados na literatura científica referentes a incidência de OS em idosos, e suas possíveis associações.

\section{METODOLOGIA}

Trata-se de uma revisão narrativa de literatura elaborada a partir de um levantamento bibliográfico de artigos científicos publicados nas bases de dados Scientific Electronic Library Online (SciELO), Biblioteca Virtual em Saúde (BVS) e Google Acadêmico. Na busca considerou-se a combinação dos seguintes descritores: obesidade sarcopênica, prevalência e idosos.

Com objetivo de obter uma amostra homogênea, selecionou-se publicações nacionais dos últimos cinco anos que fizeram análise sobre a prevalência de OS em idosos e fatores associados. Foram excluídos da seleção: artigos internacionais, estudos de revisão e os que não perfaziam o público alvo dessa revisão.

\section{RESULTADOS E DISCUSSÃO}

Foram encontrados 13 estudos realizados com idosos não institucionalizados ou de amostras de estudos populacionais, englobando quatro regiões do Brasil $(66,6 \%$ sudeste, $20 \%$ nordeste, $6,7 \%$ sul e $6,7 \%$ centro-oeste), com um total de 7.703 participantes, os quais encontram-se presentes na Tabela 1. 
Diante dos estudos apresentados identificouse uma média, considerando ambos os sexos, de prevalência de $10,7 \%$ de OS nos idosos avaliados. O resultado atual está de acordo com dados observados em estudos epidemiológicos internacionais, que encontraram uma prevalência entre 4 a $12 \%$ de OS diagnosticada através do índice de massa muscular (IMM) e 4 a $9 \%$ através do índice de massa corporal (IMC) e força de preensão palmar (CONFORTIN et al., 2019; CRISOSTOMO, 2016; NETA et al., 2018; ROCHA, 2015; ROCHA et al., 2015; ROEDIGER et al., 2017; SANTANA et al., 2019; SANTOS, 2018; SANTOS et al., 2017a; SIQUEIRA; GOBBO, 2015; STENHOLM, 2008).

Entre os estudos de Rocha (2015) e Santos (2018) observou-se que a prevalência de obesidade sarcopênica aumentou consideravelmente, de $3,3 \%$ para $10,8 \%$.
Com relação ao sexo, Rocha et al. (2015), Siqueira, Gobbo (2015), Crisostomo (2016), Almeida et al. (2017) e Confortin et al. (2019) identificaram uma maior prevalência da OS entre as mulheres. Estes resultados se assemelham ao estudo de coorte de base populacional que utilizou os dados do National Health and Nutrition Examination Survey (NHANES), nos Estados Unidos (BATSIS; VIL-LAREAL, 2018).

Com relação à idade, Rocha et al. (2015) e Confortin et al. (2019) constataram associação da maior prevalência de OS em indivíduos a partir da sétima e oitava década de vida. Este dado vai de encontro ao estudo de Batsis et al. 2017, que constatou aumento nas taxas de OS em ambos os sexos, nos indivíduos com mais de 80 anos.

Tabela 1 - Caracterização dos estudos selecionados na revisão bibliográfica. São Paulo, 2019.

\begin{tabular}{|c|c|c|c|c|c|}
\hline Autor (ano) & Local & $\begin{array}{l}\text { Amostra } \\
\text { estudada }\end{array}$ & Objetivo & $\begin{array}{l}\text { Tipo de } \\
\text { estudo }\end{array}$ & Principais resultados \\
\hline $\begin{array}{l}\text { Rocha } \\
\text { (2015) }\end{array}$ & $\begin{array}{l}\text { SP, } \\
\text { Brasil }\end{array}$ & $\begin{array}{l}1407 \text { idosos } \\
(72,8 \pm 8,13 \\
\text { anos), sendo } \\
57,1 \% \\
\text { mulheres. }\end{array}$ & $\begin{array}{l}\text { Analisar a } \\
\text { associação } \\
\text { entre OS e } \\
\text { óbito em } \\
\text { idosos } \\
\text { domiciliados } \\
\text { segundo sexo } \\
\text { e grupo etário. }\end{array}$ & $\begin{array}{l}\text { Coorte, de } \\
\text { base } \\
\text { domiciliar, que } \\
\text { utilizou dados } \\
\text { do estudo } \\
\text { SABE (Saúde, } \\
\text { Bem-Estar e } \\
\text { Envelheciment } \\
\text { o), entre } 2000 \\
\text { e } 2010 \text {. }\end{array}$ & $\begin{array}{l}\text { A prevalência de OS foi } \\
\text { de } 3,3 \% \text { e a proporção } \\
\text { óbito no período de } 10 \\
\text { anos foi de } 2,4 \% \text {. Maior } \\
\text { taxa de mortalidade no } \\
\text { grupo de idosos com } \\
\text { OS entre homens e > } \\
75 \text { anos. }\end{array}$ \\
\hline
\end{tabular}


Rocha et al. (2015)

$\begin{array}{ll}\text { SP, } & \text { Em 2000, } \\ \text { Brasil } & 1016 \text { idosos } \\ & \text { ( } \geq 70 \text { anos), } \\ & \text { sendo 59,8\% } \\ & \text { mulheres. } \\ & \text { Em 2010, } \\ & 657 \text { idosos ( } \geq \\ & 70 \text { anos), } \\ & \text { sendo 59,9\% } \\ & \text { mulheres. }\end{array}$

$\begin{array}{ll}\text { SP, } & 506 \text { idosos } \\ \text { Brasil. } & \text { (62 } \pm 9 \text { anos), } \\ & \text { sendo } 69,7 \% \\ & \text { mulheres. }\end{array}$

SP, $\quad 1373$ idosos

MG e $\quad(74,94 \pm 7,10$

$\mathrm{RN}$, anos), sendo

Brasil 64,3\%

mulheres.
Analisar a

prevalência de

OS segundo

sexo e grupos etários.

$\begin{array}{lll}\text { Santos } & \text { SP, } & 1373 \text { idosos } \\ \text { (2015) } & \text { MG e } & (74,94 \pm 7,10 \\ & \text { RN, } & \text { anos), sendo } \\ & \text { Brasil } & \begin{array}{l}64,3 \% \\ \text { mulheres. }\end{array}\end{array}$

Analisar a

associação

entre a prática

de atividade

física e a

presença de

sarcopenia ou

OS em

indivíduos com

idade $>50$

anos.

Identificar

fatores

sociodemográf

icos, as

medidas de

condição de

saúde e

desempenho

físico que se

associam à

OS em idosos

comunitários.

Coorte, de

base

domiciliar, que

utilizou dados

do estudo

SABE (Saúde,

Bem-Estar e

Envelheciment

o), entre 2000

e 2010.

Transversal.

Das 506 mulheres,

$2,5 \%$ apresentaram os.

Indivíduos

insuficientemente

ativos têm

aproximadamente 5

vezes mais chances de apresentarem OS

$(p=0,014)$.

Epidemiológic

o, transversal

Não houve diferenças

estatisticamente

e multicêntrico.

significativas entre a

amostra de OS em

relação aos idosos não

obesos e aos com

obesidade não-

sarcopênica para as

variáveis

sociodemográficas,

variáveis relacionadas

às condições de saúde

e variáveis de

desempenho físico,

exceto para a

velocidade de marcha.

Associação positiva

entre OS e

classificação de

fragilidade em idosos,

aumentando 14,2

vezes a chance de ser

pré-frágil e 112,9 vezes

a chance de ser frágil. 
Crisostomo (2016)

SP, Em 2000,

Brasil 871 idosos

$(78 \pm 5,34$

anos), sendo

$58,9 \%$

mulheres;

Em 2010,

656 idosos

$(79,5 \pm 6,59$

anos), sendo

$62,9 \%$

mulheres.

Roediger et SP, 1155 idosos

al. (2017)
$(72 \pm 9,3$

anos), sendo

$59,8 \%$

mulheres.
Santos et al. SP (2017b)
$(83,3 \pm 2,7$ anos), sendo $60 \%$ mulheres.
Neta et al. (2018)
RN, $\quad 100$ idosos

Brasil (67 \pm 8 anos), sendo $100 \%$ mulheres.
Estimar a

prevalência e a incidência de

OS em idosos

domiciliados

no município

de São

Paulo/Brasil -

2000 e 2010.

Analisar a

associação da

OS e

biomarcadores

inflamatórios

com síndrome

metabólica em

idosos.
Avaliar a

relação entre a

sarcopenia, a

capacidade

funcional e

estado

nutricional de

idosas da

comunidade.
Coorte de

idosos, de

base

domiciliar.

Utilizou dados

do Estudo

SABE (2000-

2010).

Estudo

transversal, com base nos

dados de três

coortes do

Estudo SABE

(2010).

Determinar

desfavoráveis

na composição

(obesidade,

sarcopenia ou

OS) estão

mobilidade

reduzida em

idosos com

$\geq 80$.

Transversal, com amostra

por

conveniência,

não

randomizada.$$
\text { randomizada. }
$$

à

(n)

Em 2000, 7,4\% dos idosos (6,5\% mulheres) foram identificados com OS. Em 2010, 9,2\% dos idosos $(7,2 \%$ mulheres) foram identificados com OS. Observou-se 43 novos casos de OS em 10 anos.

A prevalência de OS foi de $7,7 \%$ e de síndrome metabólica 57,9\%.

Verificou-se que mulheres com OS entre 60-69 anos, com auto avaliação do estado de saúde ruim, elevados níveis séricos de proteína C-reativa e ácido úrico estão associados a presença de síndrome metabólica.

Houve maior proporção de OS entre homens idosos $(8,6 \%)$ e maior proporção de obesidade entre idosas $(23,3 \%)$. Idosos com sarcopenia comparados àqueles sem sarcopenia tiveram 3,44 vezes mais chances de mobilidade reduzida independente do sexo e da presença de doenças osteoarticulares.

Epidemiológic 0 , observacional do tipo transversal, analítico com abordagem quantitativa.
A prevalência de OS foi de $5 \%$. Verificou-se que idosas com OS possuem pior desempenho físico. 


\begin{tabular}{|c|c|c|c|}
\hline $\begin{array}{l}\text { Santos } \\
(2018)\end{array}$ & $\begin{array}{l}\text { SP, } \\
\text { Brasil }\end{array}$ & $\begin{array}{l}\text { Em 2015, } \\
395 \text { idosos } \\
\text { ( } \geq 70 \text { anos), } \\
\text { sendo 52,8\% } \\
\text { homens; } \\
\text { Em } 2017 \text {, } \\
211 \text { idosos } \\
\text { ( } \geq 70 \text { anos), } \\
\text { sendo } 56,3 \% \\
\text { mulheres. }\end{array}$ & $\begin{array}{l}\text { Analisar, a } \\
\text { associação da } \\
\text { prática de } \\
\text { atividade física } \\
\text { e do } \\
\text { comportament } \\
\text { o sedentário } \\
\text { com } \\
\text { sarcopenia, } \\
\text { OS, OOS e } \\
\text { incapacidade } \\
\text { funcional em } \\
\text { idosos. }\end{array}$ \\
\hline
\end{tabular}

Santos et al. SP, 128 idosos (2018)

Brasil

\section{$(82,5 \pm 1,8$ \\ anos), sendo}

$63 \%$

mulheres.

Confortin et al. (2019)
SC, Brasil
477 idosos

\section{(73.2 \pm 8.8} anos), $56,8 \%$ mulheres.
Analisar a

relação entre

os

componentes

e agravos da

composição

corporal

(obesidade,

sarcopenia e

OS com a

densidade

mineral óssea

em idosos $\mathrm{cm}$

idade $\geq 80$

anos.

Identificar a

prevalência de

OS e os

fatores

associados

(sociodemográ

ficos e de

estilo de vida)

em idosos

residentes em

município do

Sul do Brasil.
Prospectivo, coorte de 24 meses.

Transversal de

base

populacional.

Transversal de

base

populacional e domiciliar.
A prevalência OS foi de $10,4 \%$, sem diferenças significativas entre os sexos. Idosos apresentaram baixa massa muscular, baixa velocidade de marcha, excesso de gordura corporal, e que permaneceram insuficientemente ativos no domínio locomoção apresentaram maior risco de OS. Mulheres com OS insuficientemente ativas nos domínios exercício físico no lazer, e locomoção apresentaram risco para incapacidade funcional.

Baixa associação entre OS e osteoporose. O risco de osteoporose em indivíduos idosos com OS mostrou-se menor do que naqueles com apenas sarcopenia. A obesidade demonstrou ser fator protetor para osteopenia/osteoporos $\mathrm{e}$, enquanto a sarcopenia como fator de risco.

A prevalência de OS foi de $4.6 \%$, não sendo identificado nenhum caso de idoso do sexo masculino com a presença do desfecho. A OS foi associada às idosas com idade $>80$ anos e que viviam sozinhas no seu domicílio. O consumo de álcool e o déficit cognitivo foram associados à ocorrência do evento. 
Nascimento,

Silva,

Prestes

(2019)

$\begin{array}{lll}\text { DF, } & 58 \text { idosos } & \text { Avaliar os } \\ \text { Brasil } & (\geq 60 \text { anos), } & \text { efeitos da Os } \\ & \text { com } & \text { na força } \\ & \text { obesidade } & \text { muscular, } \\ & \text { (gordura } & \text { função física e } \\ \text { corporal } \geq & \text { qualidade de } \\ 38 \%), \text { sendo } & \text { vida em idosas } \\ 100 \% & \text { com } \\ & \text { mulheres. } & \text { obesidade. }\end{array}$

Santana et

al. (2019)
PE

Brasil pacientes

$(71,6 \pm 7,4$

anos),

internados

em

enfermaria

de

coronariopati

as, $50,5 \%$

mulheres.
Analisar a associação entre sarcopenia e OS como preditores de prognóstico em idosos com infarto agudo do miocárdio.
Transversal.

O grupo de OS apresentou significativamente $<$ massa magra, <força de preensão manual, pontuação inferior nos testes funcionais (teste de sentar e levantar, time-up-and-go e caminhada de seis minutos), baixa aptidão física, <redução da frequência cardíaca durante a recuperação e aspecto inferior da qualidade de vida em comparação com o grupo não sarcopênico.

Retrospectivo, com coleta de dados primários e secundários. $35,4 \%$ dos indivíduos apresentaram diagnóstico de OS. Não foi encontrada associação entre OS e parâmetros de risco de doença coronariana.

Fonte: Elaborado pelos autores.

De acordo com o observado por Siqueira, Gobbo (2015) e Santos (2018) idosos insuficientemente ativos têm mais chances de apresentarem OS, demonstrando que a atividade física seja um fator fortemente associado a prevenção desta condição nestes indivíduos. Estudos recentes demostraram que uma intervenção para perda de peso combinando dieta e exercício físico em idosos com obesidade melhora a força e a qualidade muscular, além de promoverem a perda de gordura (STENHOLM, 2008).

No estudo de Santos (2015), Santos et al. (2017b), Neta et al. (2018), Santos (2018) e Nascimento, Silva, Prestes (2019) observou-se que a OS aumentou o risco de fragilidade e mobilidade reduzida em idosos, fatores estes que podem proporcionar maior incapacidade funcional e aumento no risco de quedas. Tal constatação vai de acordo ao encontrado por um compilado de estudos, onde idosos com OS apresentaram menor força, piores escores em testes de desempenho, declínio na velocidade de marcha e maior risco para desenvolver novas incapacidades (CAMPOS; LOPES; LORENÇO, 2017). 
Os episódios de queda decorrentes da OS podem levar a baixa atividade física e imobilidade em idosos, e consequentemente menor massa muscular (MACHADO, 2019). Desta forma, acabam afetando diretamente sua qualidade de vida e tornando-os mais suscetíveis a possibilidade de internação em instituições especiais, abandono e morte (NEGÓCIO, 2019).

De acordo com Roediger et al. (2017), idosos com OS que apresentam níveis séricos elevados de proteína C-reativa e ácido úrico associam-se ao aparecimento de síndrome metabólica. Relação semelhante foi encontrada por um compilado de estudos internacionais, onde contatou-se que um estado próinflamatório pode ser um dos fatores-chave na criação de um ciclo vicioso de diminuição da força muscular em idosos com obesidade (BATSIS; VILLAREAL, 2018).

No estudo de Santana et al. (2019), observou-se ausência de associação entre a OS e parâmetros de risco de doença coronariana. Tal constatação contrapõemse ao exposto pela literatura atual, visto que, idosos com OS apresentam um aumento no estresse oxidativo corporal, relacionando-se diretamente ao maior risco de desenvolvimento de doenças crônicas não transmissíveis, principalmente as cardiovasculares (TYROVOLAS et al., 2016; BELLANTI et al., 2018).

Revista Higiene Alimentar, 35 (293): e1053, 2021 ISSN 2675-0260 - DOI: 10.37585/HA2021.02incidencia

\section{CONSIDERAÇÕES FINAIS}

O presente estudo demonstrou um aumento considerável na prevalência de OS entre a população de idosos. Além disso, constatou-se que o conjunto obesidade e redução da massa muscular, estão diretamente associados ao desenvolvimento de déficits funcionais e síndrome metabólica, comprometendo a realização das atividades básicas de vida diária, qualidade de vida e saúde destes indivíduos.

Diante destes fatores e do aumento da população idosa, torna-se relevante identificar idosos com OS. É crucial promover a prevenção e o tratamento desta condição, sendo a prática de atividade física e uma alimentação adequada pontos-chave para prolongar a independência, a capacidade funcional e a condição positiva de saúde dos idosos.

Por fim, é importante salientar a realização de novos estudos com o objetivo de padronizar métodos próprios para a identificação da OS. Afinal, a partir do estabelecimento de consensos para 0 diagnóstico desta condição, aprimora-se a comparação dos achados, favorecendo à assistência à saúde do idoso.

\section{REFERÊNCIAS BIBLIOGRÁFICAS}

ALMEIDA, A. et al. Prevalência e fatores associados à polifarmácia entre os idosos residentes na comunidade. Revista Brasileira de Geriatria e Gerontologia, v. 20, n. 1, p. 143-153, 2017. 
BANN, D. et al. Physical activity across adulthood in relation to fat and lean body mass in early old age: findings from the medical research council national survey of health and development, 1946-2010. American Journal of Epidemiology, v. 179, n. 10, p. 1197-1207, 2014.

BATSIS, J.A.; VILLAREAL, D.T. Sarcopenic obesity in older adults: etiology, epidemiology and treatment strategies. Nature Reviews Endocrinology, Texas, v. 14, n. 9, p. 513-537, 2018.

BATSIS, J.A. et al. Low Lean Mass With and Without Obesity, and Mortality: Results From the 1999-2004 National Health and Nutrition Examination Survey. The journals of gerontology. Series A, Biological Sciences and Medical Sciences, v. 72 , n. 10 , p. 1445-1451, 2017.

BELLANTI, F. et al. Oxidative stress is increased in sarcopenia and associated with cardiovascular disease risk in sarcopenic obesity. Maturitas, v.109, p. 6$12,2018$.

CAMPOS, G.C; LOPES, C.S.; LOURENÇO, R.A. Obesidade sarcopênica e funcionalidade: Uma revisão da literatura. Brazilian Journal of Health and Biomedical Sciences, Rio de Janeiro, v. 16, n. 2, p. 101-108, 2017.

CRISOSTOMO L.A.H.K. Prevalência e incidência de obesidade sarcopênica em coorte de idosos domiciliados no município de São Paulo. 77p. 2016. Dissertação (Mestrado em Ciências) Faculdade de Saúde Pública, Universidade de São Paulo, São Paulo, 2016.

CRUZ-JENTOFT et al. Sarcopenia: European consensus on definition and diagnosis: Report of the European Working Group on Sarcopenia in Older People. Age and Ageing Journal, Madrid, v. 39, n. 4, p. 412-423, 2010.

CONFORTIN, S.C. et al. Obesidade sarcopênica e fatores associados em idosos residentes em um município do Sul do Brasil. Salud(i)Ciencia, v.23, p. 476481, 2019.

FERREIRA, O.G.L. et al. O envelhecimento ativo sob o olhar de idosos funcionalmente independentes. Revista da Escola de Enfermagem da USP, Paraíba, v.44, n.4, p. 1065-1069, 2010.

MACHADO, K. L.L.L. Fatores de risco para baixa massa muscular em coorte prospectiva de idosas brasileiras residentes na comunidade: São Paulo Ageing \& Health Study (SPAH). 2019. 55p. Tese (Doutorado em Ciência) Faculdade de Medicina da Universidade de São Paulo, São Paulo, 2019.

NASCIMENTO, D.C.; SILVA, C.R.; PRESTES, J. Sarcopenic obesity negatively affects muscle strength, physical function and quality of life in obese elderly women. J. Phys. Educ., Maringá, v. 30, 2019.

NETA, R.S.O. et al. Sarcopenia, funcionalidade e estado nutricional em idosas residentes na comunidade. Rev. Bras. Geriatr. Gerontol., Rio de Janeiro, v.21, n.3, p. 353-362, 2018.

NEGÓCIO, T.P. Composição corporal, força muscular e autonomia funcional de idosas. 68p. 2019. Dissertação (Mestrado em Saúde e Sociedade) Universidade do Estado do Rio Grande do Norte, Rio Grande do Norte, 2019.

ORGANIZAÇÃO MUNDIAL DA SAÚDE. Relatório mundial de envelhecimento e saúde. Genebra, Suíça, 2015.

ROCHA, M.A.P. Obesidade sarcopênica e risco para óbito em idosos brasileiros. 64p. Dissertação (Mestrado de Nutrição em Saúde Pública) - Faculdade de Saúde Pública, Universidade de São Paulo, São Paulo, 2015.

ROCHA, M.A.P. et al. Prevalência de obesidade sarcopênica em uma coorte de idosos em 2000 e 2010: estudo SABE saúde, bem-estar e envelhecimento. Nutrire, São Paulo, v. 40, p. 525, 2015. 
ROEDIGER, M.A. et al. Obesidade sarcopênica, inflamação crônica e síndrome metabólica em idosos: evidências do estudo saúde, bem-estar e envelhecimento (SABE). In: Anais 19응 Fórum Paulista De Pesquisa Em Nutrição Clínica E Experimental. Braspen Journal, São Paulo, v. 32, p. 179-180, 2017.

SAKUMA, K.; YAMAGUCHI, A. Sarcopenic obesity and endocrinal adaptation with age. Internacional Journal of Endocrinology, Nova York, p. 1-12, 2013.

SANTANA, N.M. et al. Relação entre sarcopenia e obesidade sarcopênica como preditores de prognóstico em pacientes idosos hospitalizados com infarto agudo do miocárdio. Einstein, São Paulo, v. 17, n. 4, p. 1-9, 2019

SANTOS C.M. Prevalência da obesidade, obesidade sarcopênica e fatores associados em idosos comunitários: um estudo da rede fibra. 95p. 2015. Dissertação (Mestrado em Ciências da Reabilitação) - Escola de Educação Física, Fisioterapia e Terapia Ocupacional, Universidade Federal de Minas Gerais, Belo Horizonte, 2015.

SANTOS, V.R. et al. Association of insufficient physical activity with sarcopenia and sarcopenic obesity in individuals aged 50 years or more. Revista de Nutrição, Campinas, São Paulo, v. 30, n. 2, p. 175184, 2017a.

SANTOS, V.R. et al. Obesidade, sarcopenia, obesidade sarcopênica e mobilidade reduzida em idosos brasileiros com 80 anos ou mais. Einstein, Presidente Prudente, São Paulo, v.15, n. 4, p.435-40, 2017b.

SANTOS, V.R.D. Influência da prática de atividade física e dos padrões sedentários sobre a sarcopenia, obesidade sarcopênica, obesidade osteosarcopênica e incapacidade funcional em idosos: coorte de 24 meses. 2018. 151p. Tese (Doutorado em Ciências da Motricidade, área atividade física e saúde) - Faculdade de Ciências e

Revista Higiene Alimentar, 35 (293): e1053, 2021 ISSN 2675-0260 - DOI: 10.37585/HA2021.02incidencia
Tecnologia, Universidade Estadual Paulista, Presidente Prudente, 2018.

SANTOS, V.R.D. et al. Relação entre obesidade, sarcopenia, obesidade sarcopênica e densidade mineral óssea em idosos com 80 anos ou mais. Revista Brasileira de Ortopedia, São Paulo, v.53, n.3, 2018.

SIQUEIRA, A.A., GOBBO L.A. Análise da associação da prática de atividade física com sarcopenia e obesidade sarcopênica em adultos e idosos. Revista de Educação Física de Maringá, Presidente Prudente, p. 1-17, 2015.

STENHOLM, S. et al. Sarcopenic obesity definition, etiology and consequances. Current Opinion in Clinical Nutrition and Metabolic Care, v. 11, n. 6, p. 693-700, 2008.

TYROVOLAS, S. et al. Factors associated with skeletal muscle mass, sarcopenia, and sarcopenic obesity in older adults: a multicontinent study. Journal of Cachexia, Sarcopenia and Muscle, v.7, n.3, p. 312321, 2016. 